1 Hacettepe Journal of Mathematics and Statistics

$\bigcap$ Volume 43 (3) (2014), 383-389

\title{
Coefficient bounds for certain classes of bi-univalent functions
}

\author{
B.A. Frasin *
}

\begin{abstract}
In this paper, we introduce two new subclasses of the function class $\Sigma$ of bi-univalent functions defined in the open unit disk. Furthermore, we find estimates on the coefficients $\left|a_{2}\right|$ and $\left|a_{3}\right|$ for functions in these new subclasses.
\end{abstract}

Keywords: Analytic and univalent functions, bi-univalent functions, starlike and convex functions, coefficients bounds.

2000 AMS Classification: 30C45

\section{Introduction and definitions}

Let $\mathcal{A}$ denote the class of functions of the form :

$$
f(z)=z+\sum_{n=2}^{\infty} a_{n} z^{n}
$$

which are analytic in the open unit disc $\mathcal{U}=\{z:|z|<1\}$. Further, by $\mathcal{S}$ we shall denote the class of all functions in $\mathcal{A}$ which are univalent in $\mathcal{U}$. A function $f(z)$ belonging to $\mathcal{S}$ is said to be starlike of order $\alpha$ if it satisfies

$$
\mathfrak{R}\left(\frac{z f^{\prime}(z)}{f(z)}\right)>\alpha \quad(z \in \mathcal{U})
$$

for some $\alpha(0 \leq \alpha<1)$. We denote by $\mathcal{S}^{*}(\alpha)$ the subclass of $\mathcal{S}$ consisting of functions which are starlike of order $\alpha$ in $\mathcal{U}$. Also, a function $f(z)$ belonging to $\mathcal{S}$ is said to be convex of order $\alpha$ if it satisfies

$$
\mathfrak{R}\left(1+\frac{z f^{\prime \prime}(z)}{f^{\prime}(z)}\right)>\alpha \quad(z \in \mathcal{U})
$$

for some $\alpha(0 \leq \alpha<1)$. We denote by $\mathcal{K}(\alpha)$ the subclass of $\mathcal{S}$ consisting of functions which are convex of order $\alpha$ in $\mathcal{U}$.

${ }^{*}$ Faculty of Science, Department of Mathematics, Al al-Bayt University, P.O. Box: 130095 Mafraq, Jordan Email:bafrasin@yahoo.com 
Gao and Zhou [5] showed some mapping properties of the following subclass of $\mathcal{A}$ :

$$
\mathcal{R}(\alpha, \beta)=\left\{f \in \mathcal{A}: \mathfrak{R}\left(\left(f^{\prime}(z)+\beta z f^{\prime \prime}(z)\right)>\alpha, \quad \beta>0,0 \leq \alpha<1 ; z \in \mathfrak{U}\right\} .\right.
$$

Yang and Liu [12, Theorem 3.1, p.9], proved that the class $\mathcal{R}(\alpha, \beta) \subset \mathcal{S}$ iff

$$
2(1-\alpha) \sum_{m=1}^{\infty} \frac{(-1)^{m-1}}{\beta m+1} \leq 1 .
$$

It is well known that every function $f \in \mathcal{S}$ has an inverse $f^{-1}$, defined by

$$
f^{-1}(f(z))=z \quad(z \in \mathcal{U})
$$

and

$$
f^{-1}(f(w))=w \quad\left(|w|<r_{0}(f) ; r_{0}(f) \geq \frac{1}{4}\right)
$$

where

$$
f^{-1}(w)=w-a_{2} w^{2}+\left(2 a_{2}^{2}-a_{3}\right) w^{3}-\left(5 a_{2}^{3}-5 a_{2} a_{3}+a_{4}\right) w^{4}+\cdots .
$$

A function is said to be bi-univalent in $\mathcal{U}$ if both $f(z)$ and $f^{-1}(z)$ are univalent in $\mathcal{U}$.

Let $\Sigma$ denote the class of bi-univalent functions in $\mathcal{U}$ given by (1.1). Example of functions in the class $\Sigma$ are

$$
\frac{z}{1-z}, \quad \log \frac{1}{1-z}, \quad \log \sqrt{\frac{1+z}{1-z}} .
$$

However, the familiar Koebe function is not a member of $\Sigma$. Other common examples of functions in $\mathcal{U}$ such as

$$
\frac{2 z-z^{2}}{2} \text { and } \frac{z}{1-z^{2}}
$$

are also not members of $\Sigma$.

Lewin [6] investigated the bi-univalent function class $\Sigma$ and showed that $\left|a_{2}\right|<$ 1.51. Subsequently, Brannan and Clunie [1] conjectured that $\left|a_{2}\right|<\sqrt{2}$. Netanyahu [7], on the other hand, showed that $\max _{f \in \Sigma}\left|a_{2}\right|=4 / 3$.

The coefficient estimate problem for each of the Taylor-Maclaurin coefficients $\left|a_{n}\right|(n \geq 3 ; n \in \mathbb{N})$ is presumably still an open problem.

Brannan and Taha [2] (see also [10]) introduced certain subclasses of the biunivalent function class $\Sigma$ similar to the familiar subclasses $\mathcal{S}^{*}(\alpha)$ and $\mathcal{K}(\alpha)$ (see [3]). Thus, following Brannan and Taha [2] (see also [10]), a function $f \in \mathcal{A}$ is in the class $\mathcal{S}_{\Sigma}^{*}[\alpha]$ of strongly bi-starlike functions of order $\alpha(0<\alpha \leq 1)$ if each of the following conditions are satisfied:

$$
f \in \Sigma \text { and }\left|\arg \left(\frac{z f^{\prime}(z)}{f(z)}\right)\right|<\frac{\alpha \pi}{2} \quad(0<\alpha \leq 1, z \in \mathcal{U})
$$

and

$$
\left|\arg \left(\frac{z g^{\prime}(w)}{g(w)}\right)\right|<\frac{\alpha \pi}{2} \quad(0<\alpha \leq 1, w \in \mathcal{U})
$$


where $g$ is the extension of $f^{-1}$ to $\mathcal{U}$. The classes $\mathcal{S}_{\Sigma}^{*}(\alpha)$ and $\mathcal{K}_{\Sigma}(\alpha)$ of bistarlike functions of order $\alpha$ and bi-convex functions of order $\alpha$, corresponding (respectively) to the function classes defined by (1.2) and (1.3), were also introduced analogously. For each of the function classes $\mathcal{S}_{\Sigma}^{*}(\alpha)$ and $\mathcal{K}_{\Sigma}(\alpha)$, they found non-sharp estimates on the first two Taylor-Maclaurin coefficients $\left|a_{2}\right|$ and $\left|a_{3}\right|$ (for details, see $[7,8]$ ).

The object of the present paper is to introduce two new subclasses of the function class $\Sigma$ and find estimates on the coefficients $\left|a_{2}\right|$ and $\left|a_{3}\right|$ for functions in these new subclasses of the function class $\Sigma$ employing the techniques used earlier by Srivastava et al. [9] (see also, [4] and [11] ). [8].

In order to derive our main results, we have to recall here the following lemma

1.1. Lemma. If $h \in \mathcal{P}$ then $\left|c_{k}\right| \leq 2$ for each $k$,

where $\mathcal{P}$ is the family of all functions $h$ analytic in $\mathcal{U}$ for which $\mathfrak{R} h(z)>0$ $h(z)=1+c_{1} z+c_{2} z^{2}+c_{3} z^{3}+\cdots$ for $z \in \mathcal{U}$.

\section{Coefficient bounds for the function class $\mathcal{H}_{\Sigma}(\alpha, \beta)$}

2.1. Definition. A function $f(z)$ given by (1.1) is said to be in the class $\mathcal{H}_{\Sigma}(\alpha, \beta)$ if the following conditions are satisfied:

$$
f \in \Sigma \text { and }\left|\arg \left(f^{\prime}(z)+\beta z f^{\prime \prime}(z)\right)\right|<\frac{\alpha \pi}{2} \quad(z \in \mathcal{U})
$$

and

$$
\left|\arg \left(g^{\prime}(w)+\beta w g^{\prime \prime}(w)\right)\right|<\frac{\alpha \pi}{2} \quad(w \in \mathcal{U})
$$

where $\beta>0,0<\alpha<1,2(1-\alpha) \sum_{m=1}^{\infty} \frac{(-1)^{m-1}}{\beta m+1} \leq 1$, and the function $g$ is given by

$$
g(w)=w-a_{2} w^{2}+\left(2 a_{2}^{2}-a_{3}\right) w^{3}-\left(5 a_{2}^{3}-5 a_{2} a_{3}+a_{4}\right) w^{4}+\cdots
$$

We begin by finding the estimates on the coefficients $\left|a_{2}\right|$ and $\left|a_{3}\right|$ for functions in the class $\mathcal{H}_{\Sigma}(\alpha, \beta)$.

2.2. Theorem. Let $f(z)$ given by (1.1) be in the class $\mathcal{H}_{\Sigma}(\alpha, \beta)$ where $\beta>0,0<$ $\alpha<1$, and $2(1-\alpha) \sum_{m=1}^{\infty} \frac{(-1)^{m-1}}{\beta m+1} \leq 1$. Then

$$
\left|a_{2}\right| \leq \frac{2 \alpha}{\sqrt{2(\alpha+2)+4 \beta(\alpha+\beta+2-\alpha \beta)}}
$$

and

$$
\left|a_{3}\right| \leq \frac{\alpha^{2}}{(1+\beta)^{2}}+\frac{2 \alpha}{3(1+2 \beta)} .
$$


Proof. It follows from (2.1) and (2.2) that

$$
f^{\prime}(z)+\beta z f^{\prime \prime}(z)=[p(z)]^{\alpha}
$$

and

$$
g^{\prime}(w)+\beta w g^{\prime \prime}(w)=[q(w)]^{\alpha}
$$

where $p(z)$ and $q(w)$ in $\mathcal{P}$ and have the forms

$$
p(z)=1+p_{1} z+p_{2} z^{2}+p_{3} z^{3}+\cdots
$$

and

(2.9) $\quad q(w)=1+q_{1} w+q_{2} w^{2}+q_{3} w^{3}+\cdots$.

Now, equating the coefficients in (2.6) and (2.7), we get

$$
\begin{aligned}
2(1+\beta) a_{2} & =\alpha p_{1}, \\
3(1+2 \beta) a_{3} & =\alpha p_{2}+\frac{\alpha(\alpha-1)}{2} p_{1}^{2}, \\
-2(1+\beta) a_{2} & =\alpha q_{1}
\end{aligned}
$$

and

$$
3(1+2 \beta)\left(2 a_{2}^{2}-a_{3}\right)=\alpha q_{2}+\frac{\alpha(\alpha-1)}{2} q_{1}^{2}
$$

From (2.10) and (2.12), we get

$$
p_{1}=-q_{1}
$$

and

(2.15) $8(1+\beta)^{2} a_{2}^{2}=\alpha^{2}\left(p_{1}^{2}+q_{1}^{2}\right)$.

Now from (2.11), (2.13) and (2.15), we obtain

$$
\begin{aligned}
6(1+2 \beta) a_{2}^{2} & =\alpha\left(p_{2}+q_{2}\right)+\frac{\alpha(\alpha-1)}{2}\left(p_{1}^{2}+q_{1}^{2}\right) \\
& =\alpha\left(p_{2}+q_{2}\right)+\frac{4(\alpha-1)(1+\beta)^{2}}{\alpha} a_{2}^{2} .
\end{aligned}
$$

Therefore, we have

$$
a_{2}^{2}=\frac{\alpha^{2}\left(p_{2}+q_{2}\right)}{2(\alpha+2)+4 \beta(\alpha+\beta+2-\alpha \beta)} .
$$

Applying Lemma 1.1 for the coefficients $p_{2}$ and $q_{2}$, we immediately have

$$
\left|a_{2}\right| \leq \frac{2 \alpha}{\sqrt{2(\alpha+2)+4 \beta(\alpha+\beta+2-\alpha \beta)}} .
$$

This gives the bound on $\left|a_{2}\right|$ as asserted in (2.4).

Next, in order to find the bound on $\left|a_{3}\right|$, by subtracting (2.13) from (2.11), we get

$$
6(1+2 \beta) a_{3}-6(1+2 \beta) a_{2}^{2}=\alpha p_{2}+\frac{\alpha(\alpha-1)}{2} p_{1}^{2}-\left(\alpha q_{2}+\frac{\alpha(\alpha-1)}{2} q_{1}^{2}\right)
$$


Upon substituting the value of $a_{2}^{2}$ from (2.15) and observing that $p_{1}^{2}=q_{1}^{2}$, it follows that

$$
a_{3}=\frac{\alpha^{2} p_{1}^{2}}{4(1+\beta)^{2}}+\frac{\alpha\left(p_{2}-q_{2}\right)}{6(1+2 \beta)} .
$$

Applying Lemma 1.1 once again for the coefficients $p_{1}, p_{2}, q_{1}$ and $q_{2}$, we readily get

$$
\left|a_{3}\right| \leq \frac{\alpha^{2}}{(1+\beta)^{2}}+\frac{2 \alpha}{3(1+2 \beta)} .
$$

This completes the proof of Theorem 2.2 .

Putting $\beta=1$ in Theorem 2.2, we have

2.3. Corollary. Let $f(z)$ given by (1.1) be in the class $\mathcal{H}_{\Sigma}(\alpha, 1)$ where $0<\alpha<1$, and $2(1-\alpha) \sum_{m=1}^{\infty} \frac{(-1)^{m-1}}{m+1} \leq 1$. Then

$$
\left|a_{2}\right| \leq \frac{2 \alpha}{\sqrt{2(\alpha+2)+12}}
$$

and

$$
\left|a_{3}\right| \leq \frac{9 \alpha^{2}+8 \alpha}{36}
$$

\section{Coefficient bounds for the function class $\mathcal{H}_{\Sigma}(\gamma, \beta)$}

3.1. Definition. A function $f(z)$ given by (1.1) is said to be in the class $\mathcal{H}_{\Sigma}(\gamma, \beta)$ if the following conditions are satisfied:

$$
f \in \Sigma \text { and } \mathfrak{R}\left(f^{\prime}(z)+\beta z f^{\prime \prime}(z)\right)>\gamma \quad(z \in \mathcal{U})
$$

and

$$
\mathfrak{R}\left(g^{\prime}(w)+\beta w g^{\prime \prime}(w)\right)>\gamma \quad(w \in \mathcal{U}),
$$

where $\beta>0,0 \leq \gamma<1,2(1-\gamma) \sum_{m=1}^{\infty} \frac{(-1)^{m-1}}{\beta m+1} \leq 1$, and the function $g$ is given by $(2.3)$.

3.2. Theorem. Let $f(z)$ given by (1.1) be in the class $\mathcal{H}_{\Sigma}(\gamma, \beta)$, where $\beta>0,0 \leq$ $\gamma<1$, and $2(1-\gamma) \sum_{m=1}^{\infty} \frac{(-1)^{m-1}}{\beta m+1} \leq 1$. Then

$$
\left|a_{2}\right| \leq \sqrt{\frac{2(1-\gamma)}{3(1+2 \beta)}}
$$

and

$$
\left|a_{3}\right| \leq \frac{(1-\gamma)^{2}}{(1+\beta)^{2}}+\frac{2(1-\gamma)}{3(1+2 \beta)} .
$$


Proof. It follows from (3.1) and (3.2) that there exist $p$ and $q \in \mathcal{P}$ such that

$$
f^{\prime}(z)+\beta z f^{\prime \prime}(z)=\gamma+(1-\gamma) p(z)
$$

and

$$
g^{\prime}(w)+\beta w g^{\prime \prime}(w)=\gamma+(1-\gamma) q(w)
$$

where $p(z)$ and $q(w)$ have the forms (2.8) and (2.9), respectively. Equating coefficients in (3.5) and (3.6) yields

$$
\begin{aligned}
2(1+\beta) a_{2} & =(1-\gamma) p_{1} \\
3(1+2 \beta) a_{3} & =(1-\gamma) p_{2} \\
-2(1+\beta) a_{2} & =(1-\gamma) q_{1}
\end{aligned}
$$

and

(3.10) $3(1+2 \beta)\left(2 a_{2}^{2}-a_{3}\right)=(1-\gamma) q_{2}$

From (3.7) and (3.9), we get

$$
p_{1}=-q_{1}
$$

and

(3.12) $8(1+\beta)^{2} a_{2}^{2}=(1-\gamma)^{2}\left(p_{1}^{2}+q_{1}^{2}\right)$.

Also, from (3.8) and (3.10), we find that

$$
6(1+2 \beta) a_{2}^{2}=(1-\gamma)\left(p_{2}+q_{2}\right) .
$$

Thus, we have

$$
\left|a_{2}^{2}\right| \leq \frac{(1-\gamma)}{6(1+2 \beta)}\left(\left|p_{2}\right|+\left|q_{2}\right|\right)=\frac{2(1-\gamma)}{3(1+2 \beta)}
$$

which is the bound on $\left|a_{2}^{2}\right|$ as given in (3.3).

Next, in order to find the bound on $\left|a_{3}\right|$, by subtracting (3.10) from (3.8), we get

$$
6(1+2 \beta) a_{3}-6(1+2 \beta) a_{2}^{2}=(1-\gamma)\left(p_{2}-q_{2}\right)
$$

or, equivalently,

$$
a_{3}=a_{2}^{2}+\frac{(1-\gamma)\left(p_{2}-q_{2}\right)}{6(1+2 \beta)} .
$$

Upon substituting the value of $a_{2}^{2}$ from (3.12), we obtain

$$
a_{3}=\frac{(1-\gamma)^{2}\left(p_{1}^{2}+q_{1}^{2}\right)}{8(1+\beta)^{2}}+\frac{(1-\gamma)\left(p_{2}-q_{2}\right)}{6(1+2 \beta)} .
$$

Applying Lemma 1.1 for the coefficients $p_{1}, p_{2}, q_{1}$ and $q_{2}$, we readily get

$$
\left|a_{3}\right| \leq \frac{(1-\gamma)^{2}}{(1+\beta)^{2}}+\frac{2(1-\gamma)}{3(1+2 \beta)}
$$


which is the bound on $\left|a_{3}\right|$ as asserted in (3.4).

Putting $\beta=1$ in Theorem 3.2, we have

3.3. Corollary. Let $f(z)$ given by (1.1) be in the class $\mathcal{H}_{\Sigma}(\gamma, 1)$, where $0 \leq \gamma<$ 1 , and $2(1-\gamma) \sum_{m=1}^{\infty} \frac{(-1)^{m-1}}{m+1} \leq 1$.

$$
\left|a_{2}\right| \leq \frac{1}{3} \sqrt{2(1-\gamma)}
$$

and

$$
\left|a_{3}\right| \leq \frac{(1-\gamma)(9(1-\gamma)+8)}{36}
$$

Acknowledgements. The author would like to thank the referees for their helpful comments and suggestions.

\section{References}

[1] Brannan, D. A. and Clunie, J. G. (Eds.), Aspects of Contemporary Complex Analysis Proceedings of the NATO Advanced Study Institute held at the University of Durham, Durham; July 1-20, 1979, (Academic Press, New York and London, 1980).

[2] Brannan, D. A. and Taha, T.S. On some classes of bi-univalent functions, in: S.M. Mazhar, A. Hamoui, N.S. Faour (Eds.), Mathematical Analysis and Its Applications, Kuwait; February 18-21, 1985, in: KFAS Proceedings Series, vol. 3, Pergamon Press (Elsevier Science Limited), Oxford, 1988, pp. 53-60; see also Studia Univ. Babeş-Bolyai Math. 31 (2) (1986) 70-77.

[3] Brannan, D. A., Clunie, J. and Kirwan, W. E. Coefficient estimates for a class of starlike functions, Canad. J. Math., 22, 476-485, 1970.

[4] Frasin, B. A. and Aouf, M. K New subclasses of bi-univalent functions, Appl. Math. Letters, 24, no. 9, 1569-1573, 2011.

[5] Gao, C. Y. and Zhou, S. Q. Certain subclass of starlike functions, Applied Mathematics and Computation, vol. 187, no. 1, pp. 176-182, 2007.

[6] Lewin, M On a coefficient problem for bi-univalent functions, Proc. Amer. Math. Soc. 18, 63-68, 1967.

[7] Netanyahu, E. The minimal distance of the image boundary from the origin and the second coefficient of a univalent function in $|z|<1$, Arch. Rational Mech. Anal., 32, 100-112, 1969.

[8] Pommerenke, C. Univalent functions, (Vandenhoeck and Rupercht, Göttingen, 1975).

[9] Srivastava, H. M., Mishra, A. K. and Gochhayat, P. Certain subclasses of analytic and biunivalent functions, Appl. Math. Lett., 23, 1188-1192, 2010.

[10] Taha, T. S. Topics in Univalent Function Theory, Ph.D. Thesis, University of London, 1981.

[11] Xu, Q, Gui, Y. and Srivastava, H. M. Coefficient estimates for a certain subclass of analytic and bi-univalent functions, Appl. Math. Lett., 25, 990-994, 2012.

[12] Yang, D. G. and Liu, J. L A class of analytic functions with missing coefficients, Abstract and Applied Analysis, Volume 2011, Article ID 456729, 2011. 